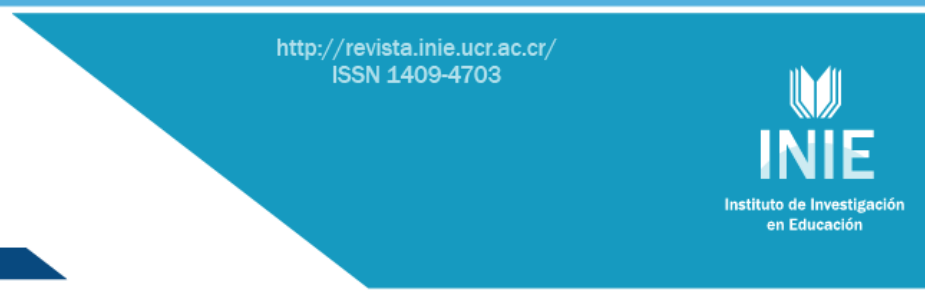

\title{
PROPIEDADES PSICOMÉTRICAS DE UNA ESCALA PARA MEDIR EMPATÍA EN ESTUDIANTES DE SECUNDARIA EN MÉXICO
}

PSYCHOMETRIC PROPERTIES OF AN SCALE EMPATHY IN JUNIOR HIGH SCHOOL STUDENTS IN MEXICO

\section{Volumen 16, Número 3 \\ Setiembre-Diciembre}

pp. $1-20$

Este número se publicó el $1^{\circ}$ de Setiembre de 2016

DOI: http://dx.doi.org/10.15517/aie.v16i3.25959

Gildardo Bautista Hernández José Ángel Vera Noriega Jesús Tánori Quintana

Ángel Alberto Valdés Cuervo

Revista indizada en REDALYC, $\underline{\text { SCIELO }}$

Revista distribuida en las bases de datos:

LATINDEX, DOAJ, E-REVIST@S, IRESIE, CLASE, DIALNET, SHERPA/ROMEO, QUALIS,

Revista registrada en los directorios:

ULRICH'S, REDIE, RINACE, OEI, MAESTROTECA, PREAL, CLACSO 


\title{
PROPIEDADES PSICOMÉTRICAS DE UNA ESCALA PARA MEDIR EMPATÍA EN ESTUDIANTES DE SECUNDARIA EN MÉXICO \\ PSYCHOMETRIC PROPERTIES OF AN SCALE EMPATHY IN JUNIOR HIGH SCHOOL STUDENTS IN MEXICO
}

\author{
Gildardo Bautista Hernández ${ }^{1}$ \\ José Ángel Vera Noriega² \\ Jesús Tánori Quintana ${ }^{3}$ \\ Ángel Alberto Valdés Cuervo ${ }^{4}$
}

\begin{abstract}
Resumen: Se realizó un estudio con enfoque cuantitativo de alcance descriptivo con el objetivo de determinar las propiedades psicométricas de una escala para medir empatía en adolescentes. De una población de 88,484 se seleccionaron mediante un muestreo no probabilístico a 2,348 estudiantes matriculados en el ciclo escolar 2014-1 correspondientes a 64 escuelas secundarias públicas de 17 municipios de Sonora, México, de las cuales 1,151 (49\%) fueron hombres y 1,197 (51\%) mujeres con una edad promedio de 13.41 años. Se encontró que la escala que mide empatía afectiva $(\alpha=.81)$ y cognitiva $(\alpha=.79)$ presenta índices de ajuste aceptables $(X 2=92.31 ; X 2 / \mathrm{gl}=$ 3.74; $\mathrm{AGFI}=.98 ; \mathrm{CVI}=.058 ; \mathrm{CFI}=.99 ; \mathrm{RMSEA}=.030 ; \mathrm{SRMR}=.016$ ). Se concluye que la escala cuenta con las propiedades psicométricas que permiten su uso para medir el constructo en adolescentes escolarizados de la región noroeste de México, no obstante es necesario realizar otros estudios para establecer diferentes evidencias de validez, tales como la convergente y la concurrente que fortalezcan las propiedades psicométricas del instrumento.
\end{abstract}

\section{Palabras clave: EMPATÍA, ADOLESCENTES, EDUCACIÓN SECUNDARIA, ESCALA PSICOMÉTRICA}

\begin{abstract}
This study was conducted with a quantitative descriptive approach aimed to determine the psychometric properties of a scale to measure empathy in adolescents. In this study 2,348 students enrolled in the 2014-1 school year were selected from a population of 88,484, using a non-probability sampling, corresponding to 64 public high school in 17 counties of Sonora, Mexico, of which 1, 151 (49\%) were men and 1, 197 (51\%) women with a mean age of 13.41 years old. It was found that the scale measuring emotional empathy $(\alpha=.81)$ and cognitive empathy $(\alpha=.79)$ has acceptable fit indices $(X 2=92.31 ; \mathrm{X} 2 \mathrm{gl}=3.74 ; \mathrm{AGFl}=.98 ; \mathrm{CVI}=.058 ; \mathrm{CFl}=.99$; $R M S E A=.030 ;$ SRMR=.016). It is concluded that the scale has satisfactory psychometric properties to measure the construct in adolescent students in the northwestern region of Mexico, however further validity studies, such as convergent and concurrent evidence, are needed in order to strengthen the psychometric properties of the instrument.
\end{abstract}

Keywords: EMPATHY, ADOLESCENTS, JUNIOR HIGH SCHOOLS, PSYCHOMETRIC SCALE

${ }_{1}^{1}$ Doctorando en el Centro de Investigación en Alimentación y Desarrollo, A.C., Sonora México. Maestría en Desarrollo regional por el Centro de Investigación en Alimentación y Desarrollo, A.C., Sonora México. Dirección electrónica: gilbher@gmail.com

2 Profesor e Investigador Titular " $D$ " en el Centro de Investigación en Alimentación y Desarrollo, A.C., Sonora México. Doctor en Psicología Social por la Universidad Nacional Autónoma de México (UNAM). D.F. México. Dirección electrónica: avera@ciad.mx

3 Profesor Investigador Auxiliar, Departamento de Educación del Instituto Tecnológico de Sonora. Sonora, México. Doctor en Ciencias Sociales, por la Universidad Autónoma de Sinaloa, Sinaloa México. Dirección electrónica: jesus.tanori@itson.edu.mx

${ }^{4}$ Profesor investigador Titular "B" del departamento de Educación del Instituto Tecnológico de Sonora. Sonora, México. Doctor en Ciencias por el Centro de Investigación en Alimentación y Desarrollo A.C., Sonora México. Dirección electrónica: angel.valdes@itson.edu.mx

Artículo recibido: 2 de setiembre, 2015

Enviado a corrección: 23 de mayo, 2016

Aprobado: 11 de julio, 2016 


\section{Introducción}

El acoso escolar es una forma de violencia entre estudiantes que se caracteriza por involucrar conductas agresivas repetitivas que se realizan con la intención de causar daño y que se presentan en el marco de relaciones con marcadas diferencias de poder entre el agresor y la víctima (Avilés Martínez y Monjas Casares, 2005; Olweus, 1993; Volk, Dane, y Marini, 2014). En las víctimas de acoso escolar se reportan problemas emocionales y conductuales, escasa integración social y pobre desempeño académico (Kochenderfer-Ladd y Troop-Gordon, 2010; O’Brennan, Bradshaw, y Sawyer, 2009). Por su parte, las estudiantes y los estudiantes agresores presentan mayor incidencia de problemas de conducta y dificultades de ajuste psicosocial que, con frecuencia, persisten en la vida adulta (Olweus, 2011; Swearer y Hymel, 2015).

No obstante que el acoso escolar es una problemática que afecta al estudiantado de todos los niveles educativos, los estudios refieren que se presenta con una alta frecuencia durante el período de la adolescencia, que abarca los años finales de la educación primaria y los estudios de secundaria (Craig Harel-Fisch, Fogel-Grinvald, Dostaler, Hetland, SimonsMorton, 2009; Ovejero, 2013). Lo anterior es consistente con los reportes de estudios realizados en México, donde se refiere que entre el $10 \%$ y el $25 \%$ de los estudiantes de secundaria se involucra en conductas agresivas hacia los pares (Castillo Rocha y Pacheco Espejel, 2008; Instituto Nacional para la Evaluación de la Educación [INEE], 2007; Valdés Cuervo y Carlos Martínez, 2014). La elevada prevalencia del acoso escolar entre estudiantes adolescentes se puede relacionar con los rápidos cambios físicos y psicosociales que ocurren en esta etapa, los cuales implican, entre otras cosas, la necesidad de afianzar su posición social y lograr aceptación entre los pares (Eslea y Rees, 2001; Espegale, Bosworth y Simon, 2000).

Si bien el acoso escolar es una problemática compleja con la cual se relacionan factores del propio estudiante y de su contexto familiar, escolar y comunitario (Postigo, González, Montoya y Ordoñez, 2013; Sung y Espegale, 2012), existe evidencia de que las emociones morales, y de forma particular la empatía se relaciona con una menor frecuencia de conductas agresivas en estudiantes adolescentes hacia sus pares (Caravita, Di Blasio y Salmivalli,, 2009; Nolasco Hernández, 2012; Stavrinides, Georgiou y Theofanous, 2010; Valdés Cuervo, Carlos Martínez, Tánori Quintana y Madrid López, en prensa). Además, se ha constado que el desarrollo de la empatía es un elemento esencial de los programas de 
prevención de la violencia escolar y la promoción de la convivencia en las escuelas (Dell y Van Schoiack, 2012; Fierro Evans, 2013; Kusché y Greenberg, 2012).

La empatía es una emoción moral que facilita a los individuos "comprender y responder de manera adaptativa a las emociones de los demás, tener éxito en la comunicación emocional, y promover la conducta prosocial" (Spreng, McKinnon, Mar y Levine, 2009, p. 62). Si bien la empatía es objeto de interés de los filósofos desde el siglo XVIII, los primeros estudios acerca del tema en la psicología se atribuyen a Titchener (1909), quien la visualiza como un concepto relacionado con los sentimientos o las emociones que se experimentan frente a los eventos emocionales de los demás (citado en Fernández Pinto, López Pérez y Márquez, 2008).

De acuerdo con Stein (1916 citado en Nolasco Hernández, 2012) en el proceso empático intervienen tres momentos: (a) la percepción de la situación del otro y sus vivencias, (b) la interiorización de la vivencia del otro y (c) la vivencia que es percibida en el otro se asume como propia. La empatía requiere de estos tres momentos, de tal modo que la finalidad del proceso no tiene por objeto el conocimiento del otro, sino la comprensión del otro.

Para Rogers (1966) la empatía es una habilidad aprendida que implica el establecimiento de un vínculo cognitivo y afectivo entre dos o más sujetos, durante el cual uno de los sujetos asume deliberadamente sensibilizarse e involucrase con la vida privada del otro. Así, se generó un nuevo enfoque que promovió una definición bidimensional de la empatía que involucra tanto las reacciones emocionales como la comprensión de un sujeto frente al estado emocional del otro. Es decir, la dimensión afectiva y cognitiva son interdependientes pues en ambos casos se trata de comprender y responder ante las experiencias problemáticas de los otros (Davis, 1983; Decety y Svetlova, 2012).

La dimensión cognitiva de la empatía implica un esfuerzo cognitivo y reflexivo del individuo que involucra tanto la toma de perspectiva, que se vincula con habilidad para entender el punto de vista de la otra persona, como la fantasía, que implica la capacidad imaginativa de la persona para ubicarse de forma ficticia en la posición del otro. Por su parte, la dimensión afectiva de la empatía se asocia con sentimientos de compasión y preocupación por las otras personas (Mestre Escrivá, Frías Navarro y Samper García, 2004; Vossen, Piotrowski y Valkenburg, 2015).

Si bien la perspectiva de la empatía como un constructo bidimensional es apoyado por autoras (Caravita et al., 2009; Decety y Svetlova, 2012; Mestre et al., 2004) y autores (Geng, 
Xia y Qin, 2012; Haro Solís, 2014; Reniers, Corcoran, Drake, Shryane y Völlm, 2011), otros investigadores (Spreng et al., 2009) e investigadoras (Hojat Gonnella, Nasca, Mangione, Veloksi y Magee, 2002) consideran la empatía como un factor unidimensional que involucra aspectos emocionales o cognitivos únicamente. En el presente estudio se parte del supuesto de que el modelo bidimensional refleja mejor la naturaleza del constructo al considerar la coexistencia de factores intelectuales y afectivos en la expresión del mismo (Fernández Pinto et al., 2008).

Una manera de estudiar las diferencias individuales en la empatía es mediante los instrumentos de autoinforme. En la literatura existen reportes de instrumentos de este tipo para medir el constructo, desarrollados por lo general en los Estados de Unidos de América y Europa (Davis, 1980; Hogan, 1969; Geng et al., 2012; Mehrabian y Epstein, 1972; Mestre et al., 2004; Reniers et al., 2011; Spreng et al., 2009). En Latinoamérica únicamente se identificaron dos publicaciones donde se reporta el desarrollo y/o evaluación de escalas para medir empatía con población adolescente (Kirst-Conceicao y Martinelli, 2014; Rey, 2003). En el caso de México únicamente se ubicó la escala multifactorial de empatía (Díaz-Loving, Andrade Palos y Nadelsticher, 1986) que fue desarrollada para población adulta.

Lo anterior constituye una dificultad para la investigación en el tema del desarrollo psicosocial, pues existe evidencia de que la empatía favorece el despliegue de habilidades sociales y el ajuste psicosocial en los adolescentes (Arsenio, 2014; Gini, Albeiro, Benelli y Altoe, 2007). Por otra parte, se reporta que los adolescentes con alta empatía tienen conductas sociales positivas: prosociales, asertivas, consideración, autocontrol, liderazgo, alto autoconcepto, alta capacidad para analizar emociones negativas, rasgos de personalidad creadora y pocas conductas sociales negativas: pasivas, agresivas, antisociales y retraimiento (Garaigordobil y García de Galdeano, 2006).

Atendiendo a la ausencia en México de instrumentos para medir la empatía, y el valor de este constructo en la comprensión del desarrollo en la adolescencia, el presente estudio se propuso diseñar y evaluar las propiedades psicométricas de un modelo de medición bidimensional de la empatía en adolescentes escolarizados. Se parte de la hipótesis de que el modelo de medición presenta una confiabilidad e índices de ajuste aceptables que lo hacen pertinente para ser utilizado con población adolescente. 


\section{Método}

\subsection{Diseño}

Se realizó un estudio de corte cuantitativo transversal de tipo no experimental donde se establecen las propiedades métricas de un modelo de medida (Kerlinger y Lee, 2002; McMillan y Schumacher, 2005).

\subsection{Participantes}

De una población total de 88,484 estudiantes matriculados en las escuelas secundarias públicas de 17 municipios de Sonora, México, durante el ciclo escolar 2014-1, se obtuvo mediante un muestreo polietápico una muestra de 2,348 estudiantes, de ambos sexos, de 64 escuelas. El procedimiento para seleccionar la unidad de análisis fue elegir a los principales municipios del Estado por su representatividad según el número de escuelas y su ubicación regional: frontera, serrana baja y alta, costa y sur. Una vez elegidos los municipios se seleccionaron, de forma no aleatoria, las escuelas y, posteriormente, de forma aleatoria ( $p=$ $.50, q=.5$ ), las aulas en las que se encontraban los estudiantes que participarían en el estudio.

Del total de estudiantes involucrados en el estudio 924 (39.35\%) cursaban el primer grado, $723(30.79 \%)$ el segundo y 701 (29.86\%) el tercero. De estos 1151 (49\%) son hombres y 1197 (51\%) mujeres, su edad promedio fue de 13.41 años $(D E=1.83$ años) con un mínimo de 11 y un máximo de 17.

La muestra se dividió de forma aleatoria en dos submuestras de similar tamaño ( $n=$ 1174). Con la primera análisis se realiza Rasch y factorial exploratorio y con la segunda, el análisis factorial confirmatorio.

\section{Instrumentos}

La escala de empatía se diseñó exprofeso para el estudio con el propósito de medir el constructo mediante 20 reactivos. Se incluyeron 15 reactivos del cuestionario de Empatía de Toronto (Spreng et al., 2009) que mide la dimensión emocional (ejemplo: Siento ternura y preocupación por los sentimientos de otros compañeros (as) menos afortunados que yo) y cinco reactivos de la escala de Davis (1983) que miden la dimensión cognitiva de la empatía (ejemplo: Puedo decir que los demás compañeros (as) están tristes aun cuando no digan nada). De los 15 ítems que evalúan la dimensión afectiva seis son ítems inversos (R2, R6, 
R9, R10, R11, R14; ver Tabla 1), mientras que de los cinco que evalúan la dimensión cognitiva dos son de tipo inverso (R18 y R20; ver Tabla 1).

Los ítems se contestaron con una escala tipo Likert con cinco opciones de respuesta 1

(nunca), 2 (casi nunca), 3 (a veces), 4 (casi siempre) y 5 (siempre). Los puntajes de los ítems inversos se recodificaron de forma tal que mayores valores indicaran mayor empatía.

Tabla 1

Especificaciones de la escala para medir empatía en adolescentes mexicanos

\begin{tabular}{|c|c|c|}
\hline Dimensión & Definición & Reactivos \\
\hline Afectiva & $\begin{array}{l}\text { Involucra sentimientos de } \\
\text { compasión y preocupación por las } \\
\text { otras personas }\end{array}$ & $\begin{array}{l}\text { R1. Cuando alguna persona se siente emocionada } \\
\text { tiendo a sentir emoción también } \\
\text { R2. Las desgracias de otras personas me molestan } \\
\text { mucho } \\
\text { R3. Me molesta ver a una persona ser tratada sin } \\
\text { respeto } \\
\text { R4. Disfruto hacer que otras personas se sientan } \\
\text { mejor } \\
\text { R5. Siento preocupación por los sentimientos de } \\
\text { otras personas menos afortunados que yo } \\
\text { R6. Cuando una persona empieza a hablar de sus } \\
\text { problemas trato de dirigir la conversación hacia } \\
\text { otras cosas } \\
\text { R7. Puedo imaginar que las personas están tristes } \\
\text { aun cuando no digan nada } \\
\text { R8. Me parece que estoy en sintonía con los } \\
\text { estados emocionales de otras personas } \\
\text { R9. No siento simpatía por las personas que se } \\
\text { producen ellos mismos los problemas } \\
\text { R10. Me siento irritado cuando alguna persona llora } \\
\text { R11. No estoy interesado en lo que sienten otras } \\
\text { personas } \\
\text { R12. Tengo un fuerte deseo de ayudar cuando veo } \\
\text { que alguna persona es molestado } \\
\text { R13. Cuando veo que alguna persona está siendo } \\
\text { tratada injustamente me siento apenado por ella } \\
\text { R14. Me parece absurdo que algunas personas } \\
\text { Iloren de alegría } \\
\text { R15. Cuando veo que se aprovechan de alguien me } \\
\text { siento protector hacia él o ella }\end{array}$ \\
\hline
\end{tabular}


Cognitiva Habilidad para entender y ubicarse en el punto de vista de la otra persona
R16. Creo que en cualquier situación existen dos versiones opuestas, entonces yo intento tomar en consideración las dos

R17. Antes de criticar a algún compañero (a), intento imaginar que sentiría si estuviera en su lugar

R18. Encuentro dificultades para ver las cosas desde el punto de vista de otros compañeros (as)

R19. En caso de desacuerdo trato de considerar los puntos de vista del otro compañero (a)

R20. Si me siento bien acerca de algo que hago, no pierdo el tiempo escuchando otras razones de otros

Fuente: Elaboración propia

\section{Procedimiento}

Los ítems fueron traducidos por dos expertos en el idioma inglés y dominio técnico del área de la psicología. Posteriormente se indagó acerca de la evidencia de la validez de contenido de la escala con expertos en el tema y se verificó la sensibilidad y comprensión de los reactivos con un grupo de estudiantes de esta edad.

Una vez elaborada la versión preliminar del instrumento se explicó a directivas, directivos y docentes de las mismas el objetivo del estudio y se solicitó autorización para acceder al estudiantado. Participaron en el estudio los alumnos, de ambos sexos, cuyos padres y madres dieron consentimiento informado por escrito para que contestaran el instrumento.

Posteriormente, se pidió a los estudiantes su participación voluntaria garantizándoles la confidencialidad de la información. Durante la aplicación se entregó un ejemplar de la escala con una hoja de respuesta electrónica a cada estudiante; luego, se leyó el objetivo de la investigación y se explicó el contenido del instrumento y la forma de relleno de la hoja de respuesta. La administración se realizó durante el horario de clases de cada grupo en una sola sesión. A medida que el alumnado entregaba sus formularios de respuesta, se verificó que los datos de identificación y todas las preguntas estuviesen contestados correctamente para evitar problemas al momento de capturar la información en el lector óptico.

Se utilizaron el análisis de crédito parcial, un análisis factorial exploratorio y otro confirmatorio. El modelo de crédito parcial de Mesters (1982) permite valorar la productividad y la unidimensionalidad de los reactivos. Para considerar a un reactivo productivo y con buen ajuste se tomaron como referencia los parámetros e índices establecidos por Linacre (2008) y González Montesinos (2008). Los parámetros se evaluaron en su conjunto y se decidió 
excluir aquellos reactivos que no cumplieran con dos de los parámetros para análisis posteriores (ver Tabla 2).

Tabla 2

Parámetros, definición y valores para considerar a un reactivo productivo y con ajuste en el modelo

\begin{tabular}{|c|c|c|}
\hline Parámetros & Definición & Valores productivos \\
\hline MEASURE & $\begin{array}{l}\text { Es la calibración de dificultad del reactivo } \\
\text { expresada en rango de } 0 \text { a } 100\end{array}$ & $\begin{array}{l}50 \text { indica el rasgo medio del sujeto } \\
\text { Valores superiores significan } \\
\text { mayor dificultad y valores menores } \\
\text { menor dificultad }\end{array}$ \\
\hline INFIT MSQ & $\begin{array}{l}\text { Indica el ajuste interno del reactivo al modelo y es } \\
\text { sensible al comportamiento inesperado que afecta } \\
\text { a los reactivos cuya dificultad está cerca del nivel } \\
\text { de habilidad o rasgo de una persona }\end{array}$ & 0.5 a 1.5 \\
\hline $\begin{array}{l}\text { OUTFIT } \\
\text { MSQ }\end{array}$ & $\begin{array}{l}\text { Ajuste interno sensible al comportamiento } \\
\text { inesperado que afecta a los reactivos cuya } \\
\text { dificultad está lejos del nivel de habilidad de una } \\
\text { persona }\end{array}$ & 0.5 a 1.5 \\
\hline PTBIS & $\begin{array}{l}\text { Indica la correlación punto biserial entre cada } \\
\text { reactivo calificado dicotómicamente y la puntuación } \\
\text { total observada para el reactivo }\end{array}$ & $\begin{array}{l}\text { Entre más se incremente el valor } \\
\text { de CORR mayor la indicación de } \\
\text { unidimensionalidad en la escala }\end{array}$ \\
\hline DISCR & $\begin{array}{l}\text { Indica el potencial de cada reactivo a distinguir } \\
\text { sustentantes de baja y alta habilidad o rasgo }\end{array}$ & $\begin{array}{l}\text { La expectativa del modelo es } 1 \text { sin } \\
\text { rebasar el umbral de } .90 \text { inferior y } \\
1.20 \text { superior }\end{array}$ \\
\hline
\end{tabular}

Fuente: Elaboración propia a partir de Linacre (2008) y González (2008).

Posteriormente, las medidas fueron sometidas a un análisis factorial exploratorio con el objetivo de valorar la dimensionalidad de la escala. Dado que se verificó el supuesto de normalidad univariante de las variables, se utilizó el método de extracción de máxima verosimilitud y rotación Oblimin, ya que se supone relación entre los puntajes de las escalas (Cea D'Ancona, 2004; Hair, Anderson, Tatham y Black, 1999).

Finalmente, se realizó un análisis factorial confirmatorio con el método de estimación de máxima verosimilitud, para evaluar el ajuste del modelo de medida se utilizaron los índices propuestos por Hair, Black, Barbin y Anderson (2010): ji-cuadrado $\left(X^{2}\right)$, la razón entre X2 y el número de grados de libertad $\left(\mathrm{X}^{2} / \mathrm{gl}\right)$, raíz del residuo cuadrático promedio $(R M R)$ y raíz del residuo cuadrático promedio de aproximación (RMSEA), índice de bondad de ajuste corregido $(A G F I)$, índice de bondad de ajuste comparativo $(C F)$ y el índice de validación 
cruzada esperada (ECVI). El análisis de crédito parcial se realizó con apoyo del Winesteps; el programa estadístico IBM SPSS 20 se utilizó para el análisis factorial exploratorio y el AMOS 20 para el factorial confirmatorio.

\section{Resultados}

\subsection{Validez de contenido}

Los reactivos fueron evaluados por dos jueces y una jueza expertos con experiencia en investigación en la temática, experiencia en psicometría y en trabajo con adolescentes. Se encontró un $100 \%$ de concordancia en la valoración de los mismos acerca de la pertinencia de los reactivos para medir el constructo. Únicamente sugirieron correcciones menores de redacción que fueron realizadas.

Posteriormente se administró la prueba a un grupo de estudiantes de secundaria y se les pidió que evaluaran la comprensibilidad de la redacción de los ítems y su pertinencia para su grupo de edad. A partir de sus respuestas se realizaron correcciones a la redacción de dos ítems.

\subsection{Dimensionalidad y confiabilidad. Análisis de criterio parcial}

Los resultados de esta prueba sugieren la existencia de una estructura bidimensional en la escala. A su vez, cada uno de los factores forma una estructura unidimensional. Del análisis realizado para la subescala de empatía afectiva se obtuvo una fiabilidad de .99; sin embargo, se encontró que los reactivos: R10 (INFIT= 2.90; OUTFIT= 4.85; PBSE= -.56, $D I S C R I M=-2.54)$ y $\mathrm{R} 14(P B S E=.25, D I S C R I M=.55)$ presentaron problemas al no ajustarse dentro de los parámetros establecidos, por lo que se omitieron en los análisis siguientes. Referente a la subescala de empatía cognitiva, se obtuvo una fiabilidad global de .99; además, todos los reactivos se ajustaron dentro de los parámetros establecidos, por lo que se mantuvieron en los análisis siguientes (ver Tabla 3). 
Tabla 3

Estimación de parámetros de los reactivos de la escala de empatía afectiva y cognitiva

\begin{tabular}{|c|c|c|c|c|c|c|}
\hline Dimensión & $\begin{array}{c}\text { Reactiv } \\
0\end{array}$ & $\begin{array}{c}\text { MEASUR } \\
E\end{array}$ & $\begin{array}{c}\text { IN.MS } \\
Q\end{array}$ & $\begin{array}{c}\text { OUT.MS } \\
Q\end{array}$ & $\begin{array}{c}P B S \\
E\end{array}$ & $\begin{array}{c}\text { DISCRI } \\
M\end{array}$ \\
\hline \multirow[t]{15}{*}{ Afectiva } & $\mathrm{R} 1$ & 49.01 & .84 & .85 & .59 & 1.24 \\
\hline & $\mathrm{R} 2$ & 52.78 & .89 & .87 & .55 & 1.21 \\
\hline & R3 & 45.64 & .73 & .72 & .67 & 1.40 \\
\hline & $\mathrm{R} 4$ & 44.22 & .71 & .69 & .68 & 1.32 \\
\hline & $\mathrm{R} 5$ & 47.42 & .67 & .66 & .71 & 1.57 \\
\hline & $\mathrm{R} 6$ & 54.60 & 1.06 & 1.02 & .42 & 0.93 \\
\hline & $\mathrm{R} 7$ & 50.20 & .82 & .79 & .61 & 1.33 \\
\hline & $\mathrm{R} 8$ & 52.17 & .77 & .75 & .63 & 1.38 \\
\hline & $\mathrm{R} 9$ & 54.08 & .98 & .94 & .48 & 1.06 \\
\hline & $\mathrm{R} 10^{*}$ & 46.81 & 2.90 & 4.85 & -.56 & -2.54 \\
\hline & $\mathrm{R} 11$ & 53.71 & 1.13 & 1.08 & .39 & 0.85 \\
\hline & $\mathrm{R} 12$ & 47.48 & .71 & .69 & .68 & 1.52 \\
\hline & $\mathrm{R} 13$ & 48.23 & .72 & .71 & .68 & 1.50 \\
\hline & $\mathrm{R} 14^{*}$ & 54.67 & 1.35 & 1.36 & .25 & .55 \\
\hline & $\mathrm{R} 15$ & 50.68 & .78 & .76 & .63 & 1.38 \\
\hline \multirow[t]{5}{*}{ Cognitiva } & $\mathrm{R} 16$ & 52.52 & .92 & .92 & .61 & 1.09 \\
\hline & $\mathrm{R} 17$ & 46.80 & .87 & .87 & .63 & 1.17 \\
\hline & $\mathrm{R} 18$ & 55.33 & 1.10 & 1.07 & .53 & .88 \\
\hline & $\mathrm{R} 19$ & 49.39 & .83 & .82 & .66 & 1.22 \\
\hline & $\mathrm{R} 20$ & 52.39 & 1.29 & 1.29 & .46 & .62 \\
\hline
\end{tabular}

Nota. * =reactivos fuera de los rangos aceptables de ajuste

Fuente: Elaboración propia

\subsubsection{Análisis de la estructura interna del instrumento}

Análisis factorial exploratorio. Con los 13 reactivos que resultaron con valores de ajuste aceptables en el análisis previo para la subescala de empatía afectiva se llevó a cabo el análisis factorial exploratorio, utilizando el método de extracción de máxima verosimilitud y rotación Oblimin. Los resultados de la prueba de esfericidad de Bartlett $\left(\left.\right|^{2}=11655.18, p<\right.$ $.000)$ y el valor de Kaiser-Meyer-Olkin (KMO), de .93, indican la adecuación de la solución 
factorial (Cea D’Ancona, 2004; Martínez Arias, Hernández Lloreda y Hernández Lloreda, 2006).

No se incluyeron en el modelo los reactivos: $\mathrm{R} 6, \mathrm{R} 9$ y $\mathrm{R} 11$ por obtener comunalidades menores a .30 (Hair et al., 1999; Martínez et al., 2006). Los reactivos tienen una estructura unidimensional y explican el $49.43 \%$ de la varianza común (ver Tabla 4).

Tabla 4

Peso factorial y comunalidades de la subescala empatía emocional

\begin{tabular}{lcc}
\hline & \multicolumn{2}{l}{ Factor } \\
\cline { 2 - 3 } & $\begin{array}{c}\text { Peso } \\
\text { factorial }\end{array}$ & $h^{2}$ \\
& .79 & .63 \\
\hline $\begin{array}{l}\text { R5. Siento preocupación por los sentimientos de otros compañeros } \\
\text { (as) menos afortunados que yo }\end{array}$ & .78 \\
\hline R3. Me molesta ver a algún compañero (a) ser tratado sin respeto & .61 \\
$\begin{array}{l}\text { R4. Disfruto hacer que otros compañeros (as) se sientan mejor } \\
\text { R12. Tengo un fuerte deseo de ayudar cuando veo que algún }\end{array}$ & .61 \\
$\begin{array}{l}\text { compañero(a) es molestado } \\
\text { R13. Cuando veo que algún compañero (a) está siendo tratado (a) } \\
\text { injustamente me siento apenado por él (ella) }\end{array}$ & .76 \\
$\begin{array}{l}\text { R15. Cuando veo que se aprovechan de alguien me siento protector } \\
\text { hacia él o ella }\end{array}$ & .73 \\
$\begin{array}{l}\text { R8. Me parece que estoy en sintonía con los estados emocionales de } \\
\text { otros compañeros (as) }\end{array}$ & .64 \\
$\begin{array}{l}\text { R1. Cuando algún compañero (a) se siente emocionado tiendo a } \\
\text { sentirme emocionado también } \\
\text { R7. Puedo imaginar que los demás compañeros (as) están tristes aun } \\
\text { cuando no digan nada } \\
\text { R2. Las desgracias de otros compañeros (as) me molestan mucho }\end{array}$ & .64 \\
\hline
\end{tabular}

Nota. $h^{2}=$ comunalidades

Fuente: Elaboración propia

Asimismo, con los cinco reactivos que resultaron con valores de ajuste aceptables, en el análisis previo de la subescala empatía cognitiva se llevó a cabo el análisis factorial exploratorio utilizando el método de extracción de máxima verosimilitud y rotación Oblimin. Se obtuvo un índice de $K M O$ de .78 y una prueba de esfericidad de Bartlett significativa $\left(\left.\right|^{2}=\right.$ $2784.90, p<.000)$, los cuales indican la adecuación de la solución factorial. 
Se eliminó el ítem R20 por tener una comunalidad menor a .30 (Hair et al., 1999; Martínez et al., 2006). Los reactivos conforman una estructura unidimensional y explican el 49.95\% de la varianza común (ver Tabla 5).

\section{Tabla 5}

\section{Peso factorial y comunalidades de la subescala empatía cognitiva}

\begin{tabular}{lcc}
\hline & Factor \\
& 1 & $h^{2}$ \\
& .578 \\
$\begin{array}{l}\text { R17. Antes de criticar a algún compañero (a) intento imaginar que sentiría } \\
\text { si estuviera en su lugar }\end{array}$ & .760 & .577 \\
$\begin{array}{l}\text { R19. En caso de desacuerdo trato de considerar los puntos de vista del } \\
\text { otro compañero (a) }\end{array}$ & .759 & .532 \\
$\begin{array}{l}\text { R16. Creo que en cualquier situación existen dos versiones opuestas, } \\
\text { entonces yo intento tomar en consideración las dos }\end{array}$ & .730 & \\
$\begin{array}{l}\text { R18. Encuentro dificultades para ver las cosas desde el punto de vista de } \\
\text { otros compañeros (as) }\end{array}$ & .558 & .311 \\
\hline
\end{tabular}

$h^{2}=$ Comunalidad

Fuente: Elaboración propia

En el análisis factorial confirmatorio se empleó el método de estimación de máxima verosimilitud para determinar la bondad de ajuste empírico del modelo. El modelo inicial presentó problemas de ajuste, por lo que se prosiguió a su adecuación a través de los valores de significación y los índices de modificación de los parámetros individuales y que informan de los problemas de ajuste existentes en los datos (Cea, 2004).

En este sentido se eliminaron cinco reactivos para alcanzar una $\left.\right|^{2}=49.41$ con un $p$ valor asociado mayor a .000, lo que indica un ajuste adecuado del modelo (Hair et al., 1999). Los valores de los demás índices indican el ajuste del modelo a los datos, por lo que en el caso de la empatía afectiva, con cinco reactivos, representa un modelo empíricamente sustentable (Cea D'Ancona, 2004; Hair et al., 2010). En el caso de la empatía cognitiva se obtuvo un ajuste general satisfactorio con los reactivos, por lo que se puede afirmar que con los cuatro reactivos no existen diferencias entre las matrices de varianzas-covarianzas observadas contra el modelo propuesto. Además, todos los índices de ajuste analizados resultaron adecuados, por lo que se puede asumir el modelo propuesto como empíricamente sustentable. Finalmente se realizó un modelo con las dos dimensiones: empatía afectiva y cognitiva, alcanzando valores de ajuste satisfactorios en: $\mathrm{X}^{2} / g l=3.74 ; C F I=.99 ; R M S E A=.038$ 
y $S R M R=.016$, no así en $\left.\right|^{2}=92.31$ estadístico considerado susceptible al tamaño de la muestra (Hair et al., 1999), por lo que se puede asumir el modelo propuesto como empíricamente sustentable (ver Tabla 6).

Tabla 6

Índices de ajuste de los modelos para la empatía y sus dimensiones

\begin{tabular}{lcccccccc}
\hline Modelo & $g l$ & $X^{2}$ & $X^{2} / g l$ & AGFI & ECVI & CFI & RMSEA & SRMR \\
\hline Empatía afectiva inicial & 35 & 865.15 & 24.71 & .88 & .38 & .92 & .100 & .043 \\
Empatía afectiva final & 5 & 49.41 & 9.88 & .97 & .012 & .98 & .061 & .019 \\
Empatía cognitiva & 2 & 2.788 & 1.39 & .99 & .008 & 1.00 & .013 & .005 \\
Empatía & 26 & 92.31 & 3.74 & .98 & .058 & .99 & .030 & .016 \\
\hline
\end{tabular}

Fuente: Elaboración propia

El modelo de análisis factorial confirmatorio asume que existe una relación de covarianza entre los dos tipos de empatía: afectiva y cognitiva. Además, los reactivos asociados a cada una de las dimensiones tienen pesos de regresión que nos permiten indicar que todos ellos son igualmente importantes, dados sus valores encontrados y la variación entre ellos (ver Figura 1). 


\section{Figura 1}

Resultados del modelo de dos dimensiones para medir empatía en adolescentes

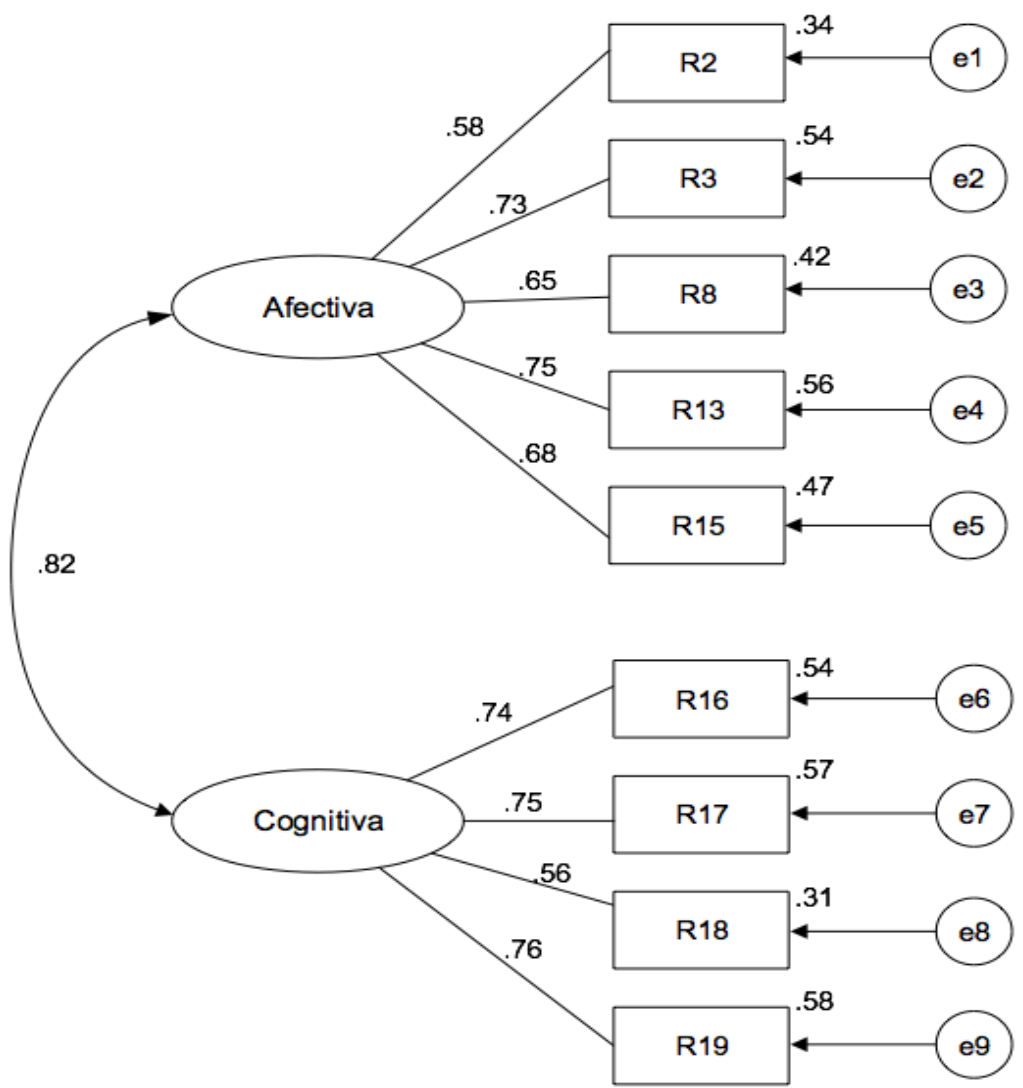

Nota. Los números dentro de los rectángulos corresponden al número de reactivo de cada una de las sub-escalas.

Fuente: Elaboración propia

\subsection{Análisis de confiabilidad}

Se realizó un análisis la confiabilidad mediante el análisis de la consistencia interna de los puntajes de las subescalas y globales con el estadístico Alfa de Cronbach. Los resultados sugieren una adecuada consistencia interna en todos los casos (Hair et al., 1999; ver Tabla 7).

Tabla 7

Coeficientes de confiabilidad de la escala y subescalas

\begin{tabular}{lcccc}
\hline & & Hombres & Mujeres & Total \\
\hline Empatía & 9 reactivos & .88 & .84 & .87 \\
Afectiva & 5 reactivos & .82 & .77 & .81 \\
Cognitiva & 4 reactivos & .82 & .74 & .79 \\
\hline
\end{tabular}

Fuente: Elaboración propia 


\section{Conclusiones}

De los resultados de los análisis se infiere que el instrumento desarrollado para medir empatía con base en las propuestas teóricas de Davis (1983) y Spreng et al. (2009) presenta propiedades psicométricas que lo hacen pertinente para medir este constructo en adolescentes del noroeste de México. Esto abre la posibilidad de evaluar dicha variable como un factor asociado al acoso escolar en México y en la región, tal y como se ha propuesto en la literatura (Van Noorden, Haselager, Cillessen y Bukowski, 2014)

Los resultados sugieren la pertinencia de un modelo bidimensional de medición de la empatía donde se integren aspectos cognitivos y afectivos (Davis, 1983; Haro Solís, 2014; Mestre Escrivá et al., 2004). Lo anterior permite afirmar que a través de este instrumento es posible analizar la reacción afectiva y cognitiva de un sujeto frente al estado emocional del otro (Davis, 1983), pero también que cada dimensión se ajusta a la unidimensionalidad y puede servir para estimar por separado la capacidad explicativa de estas habilidades (Davis, 1980) o cuando se opte por analizar y profundizar en una dimensión especifica (Spreng et al., 2009).

La escala permite evaluar tanto la habilidad de adolescentes para comprender el punto de vista de la otra persona como los sentimientos que denotan empatía hacia los otros: compasión y preocupación ante el malestar de otros (Davis, 1980; Decety y Svetlova, 2012). En este sentido se tiene una escala que, además de ajustarse a un modelo sustentable con técnicas estadísticas robustas, se trata de una escala con parsimonia y pertinencia cultural (Reyes Lagunes, 1993) para niños y adolescentes en el ecosistema mexicano y que resuelve un vacío en el tema (Haro Solís, 2014).

Se concluye que la escala posee propiedades psicométricas que hacen factible su uso en la evaluación e investigación acerca del constructo en adolescentes mexicanos escolarizados. La información que aporta la escala puede resultar valiosa para los programas de prevención de la violencia escolar que consideren dentro de sus acciones el desarrollo en el estudiantado de la toma de perspectiva y la preocupación empática por los pares (Gorostiaga, Balluerka y Soroa, 2014).

Se sugiere reforzar el análisis de las propiedades psicométricas de la escala indagando en su evidencia de validez concurrente mediante su relación con los reportes de agresión en estudiantes (Nolasco Hernández, 2012; Stavrinides et al., 2010) o con los roles que asume el estudiantado en situaciones de violencia escolar; esta es una problemática presente en las escuelas mexicanas que exige encontrar respuestas para desarrollar un modelo de 
explicación del fenómeno (Haro, 2014). También, es necesario determinar si el cuestionario presenta invarianza factorial en ambos géneros, en los estudiantes de distintas regiones, condiciones socioeconómicas y etnias. Esto, sin lugar a dudas, ofrece información acerca de la pertinencia de utilizar los resultados de la escala con diferentes grupos de adolescentes.

\section{Referencias}

Arsenio, William. (2014). Moral emotion attributions and aggression. En Killen, Melanie y Smetana, Judith (Eds.). Handbook of moral development ( $2^{\text {nd }}$ Ed., pp. 235-255). New York: Psychology Press.

Avilés, José y Monjas, Inés. (2005). Estudio de incidencia de la intimidación y el maltrato entre iguales en la educación secundaria obligatoria mediante el cuestionario CIMEI (Avilés, 1999) -Cuestionario sobre Intimidación y Maltrato entre Iguales-. Anales de Psicología, 21(1), 27-41. Recuperado de http://revistas.um.es/analesps/article/view/27 $\underline{091 / 26281}$

Caravita, Simona, Di Blasio, Paola y Salmivalli, Christina. (2009). Unique and interactive effects of empathy and social status on involvement in bullying. Social Development, 18, 140-163. doi:10.1111/j.1467-9507.2008.00465.x

Castillo, Carmen y Pacheco, Maria (2008). Perfil del maltrato (bullying) entre estudiantes de secundaria en la ciudad de Mérida, Yucatán. Revista Mexicana de Investigación Educativa, 13(38), 825-842.

Cea, María (2004). Análisis multivariable. Teoría y práctica en la investigación social (2 ${ }^{\mathrm{da}}$ Ed.). Madrid: Síntesis.

Craig, Wendy, Harel-Fisch, Yossi, Fogel-Grinvald, Haya, Dostaler, Suzanne, Hetland, Jorn, Simons-Morton, Bruce. (2009). A cross-national profile of bullying and victimization among adolescent in 40 countries. International Journal of Public Health, 54, 216-224. doi:10.1007/s 00038-00 9-5413-9

Davis, Mark H. (1980). A multidimensional Approach to Individual Differences in Empathy. JSAS Catalog of Selected Documents in Psychology, 10, 85. Recuperado de http://www.uv.es/ friasnav/Davis 1980.pdf

Davis, Mark. H. (1983). Measuring individual differences in empathy: Evidence for a multidimensional approach. Journal of Personality and Social Psychology, 44, 113-126. doi:10.1037/0022-3514.44.1.113

Decety, Jean y Svetlova, Margarita. (2012). Putting together phylogenetic and ontogenetic perspectives on empathy. Developmental Cognitive Neuroscience, 2, 1-24. doi:10.1016/j.den.2011.05.003

Dell, Pam y Van Schoiack, Leithua (2012). Social and emotional skill training with second step: Violence prevention curriculum. En Jimerson, Shane; Nickerson Amanda; Mayer, 
Mathew y Furlong, Michael (Eds.). Handbook of school violence and safety (pp. 423433). New York: Routledge.

Díaz, Rolando, Andrade, Patricia y Nadelsticher, Abraham. (1986). Desarrollo de la escala multidimensional de empatía. Revista de Psicología Social y Personalidad, 2(1), 1-12.

Eslea, Mike y Rees, Josette. (2001). At what age are children more likely to be bullied at school? Aggressive Behavior, 27, 419-429. doi:10.1002/ab.1027

Espegale, Dorothy, Bosworth, Kris y Simon, Thomas. (2000). Examining the social context for bullying behavior in early adolescente. Journal of Counseling and Development, 78, 326-333. doi:10.1002/j.1556-6676.2000.tb01914.x

Fernández Pinto, Irene; López Pérez, Belén y Márquez, María. (2008). Empatía: Medidas, teorías y aplicaciones en revisión. Anales de Psicología 24(2), 284-298.

Fierro Evans, María Cecilia. (2013). Convivencia inclusiva y democrática. Una perspectiva para gestionar la seguridad escolar. Sinéctica, 40. Recuperado de http://www. sinectica.iteso.mx/articulo/id=40 convivencia inclusiva y democratica una perspectiva para gestionar la seguridad escolar

Garaigordobil, Maite y García, Patricia. (2006). Empatía en niños de 10 a 12 años. Psicothema. Psicothema, 18(2), 180-186.

Geng Yaoguo, Xia, Dan y Qin, Beibei (2012). The Basic Empathy Scale: A Chinese Validation of a Measure of Empathy in Adolescents. Child Psychiatry Human Development, 43, 499-510. doi:10.1007/s10578-011-0278-6

Gini, Gianluca, Albeiro, Paolo, Benelli, Beatrice y Altoe, Gianmarco. (2007). Does empathy predict adolescents' bullying and defending behavior? Aggressive Behavior, 33, 467476. doi:10.1002/ab. 20204

González, Manuel (2008). El análisis de reactivos con el modelo Rach. México: Universidad de Sonora/Instituto Nacional para la Evaluación de la Educación.

Gorostiaga, Arantxa, Balluerka, Nekane y Soroa, Goretti. (2014). Evaluación de la empatía en el ámbito educativo y su relación con la inteligencia emocional. Revista de Educación, 364, 12-38. doi:10.4438/1988-592X-RE-2014-364-253

Hair, Joseph F., Anderson, Rolph E., Tatham, Ronald L. y Black, William C. (1999). Análisis multivariante $\quad\left(5^{\text {ta }} \quad\right.$ ed. $)$ Madrid: Prentice

Hair, Joseph F., Black, William C., Babin, Barry J. y Anderson, Rolph E. (2010). Multivariate data analysis ( $7^{\text {th }}$ ed.). New Jersey: Pearson Prentice Hall.

Haro Solís, Israel. (2014). Factores vinculados con el papel que asumen los alumnos involucrados en el maltrato entre iguales (Bullying). (Tesis doctoral inédita), Universidad Nacional Autónoma de México, Ciudad de México. 
Hogan, Robert. (1969). Development of an empathy scale. Journal of Consulting and Clinical Psychology, 33, 307-316. doi:10.1037/h0027580

Hojat, Mohammadreza, Gonnella, Joseph S., Nasca, Thomas J., Mangione, Salvatore, Veloksi, J. Jon y Magee, Michael. (2002). The Jefferson Scale of Physician Empathy: Further psychometric data and differences by gender and specialty at item level. Academic Medicine, 77(10), 58-60. Recuperado de http://journals. Iww.com/academicmedicine/Citation/2002/10001/The Jefferson Scale 0 f Physician Empathy Further.19.aspx

Instituto Nacional para la Evaluación de la Educación (2007). Disciplina, violencia y consumo de sustancias nocivas a la salud en escuelas primarias y secundarias mexicanas. México: INEE.

Kerlinger, Fred y Lee, Howard B. (2002). Investigación del comportamiento. Métodos de investigación en Ciencias Sociales ( ${ }^{\text {ta }}$ Ed.). México: McGraw Hill.

Kirst-Conceicao, Andrea da Conha y Martinelli, Selma de Casiá (2014). Análisis psicométricas iniciais de uma escala de empatía infantojuvenil (EEempa-IJ). Avaliacao Psicológica, 13(3), 351-358. Recuperado de http://pepsic.bvsalud.org/pdf/avp/v13n3/v13n3a07.pdf

Kochenderfer-Ladd, Becky y Troop-Gordon, Wendy. (2010). Introduction to the special issue contexts, causes, and consequences. New directions in peer victimization research. Merrill-Palmer Quarterly, 56, 221-230. doi:10.1353/mpq.0.0048

Kusché, Carol y Greenberg, Mark (2012). The PATHS curriculum: Promoting emotional literacy, prosocial behavior, and caring classroom. En Jimerson, Shane; Nickerson Amanda; Mayer, Mathew y Furlong, Michael (Eds.), Handbook of school violence and safety (pp. 435-446). New York: Routledge.

Linacre, John Michael. (2008). Winsteps: Rasch measurement computer program. Chicago: Winsteps.

Martínez Arias, María, Hernández, María y Hernández, María V. (2006). Psicometría. Madrid: Alianza Editorial.

Mesters, Georp. (1982). A Rasch model for partial credit scoring. Psychometrika, 47(2), 149174. Recuperado de http://www3.nccu.edu.tw/ mnyu/Study\%20of\%20Test\%20 Theory/ A\%20Rasch\%20model\%20for\%20partial\%20credit\%20scoring.pdf

McMillan, James y Schumacher, Sally (2005). Investigación educativa (5 $5^{\text {ta }}$ Ed.). Madrid, España:

Pearson.

Mestre Escrivá, María Vicenta, Frías Navarro, María Dolores y Samper García, Paula. (2004). La medida de la empatía: análisis del Interpersonal Reactivity Index. Psicothema, 16(2), 255-260.

Mehrabian, Albert y Epstein, Albert (1972). A measure of emotional empathy. Journal of Personality, 40(4), 525-543. doi:10.1111/j.1467.6494.tb00078.x 
Nolasco Hernández, Alberto (2012). La empatía y su relación con el acoso escolar. REXE. Revista de Estudios y Experiencias en Educación, 11(22), 35-54. Recuperado de http://www.redalyc.org/pdf/2431/243125410002.pdf.

O'Brennan, Lindsey, Bradshaw, Catherine y Sawyer, Anna (2009). Examining developmental differences in the social emotional problems among frequent bullies, victims, and bully/victims. Psychology in the Schools, 46 (2), 100-115. doi:10.1002/pits.20357

Olweus, Dan (1993). Bullying at school. What we know and what we can do. Oxford: Blackwell.

Olweus, Dan (2011). Bullying at school and later criminality: Finding from three Swedish community samples of males. Criminal Behaviour and Mental Health, 21, 151-156. doi:10.1002/cbm.806

Ovejero, Anastasio (2013). El acoso escolar: cuatro décadas de investigación internacional. En Ovejero, Anastasio, Smith, Peter y Yubero, Santiago (Eds.). El acoso escolar y su prevención. Perspectivas internacionales (pp. 11-56). Madrid: Biblioteca Nueva.

Postigo, Silvia, González, Remedios, Montoya, Inmaculada y Ordoñez, Ana. (2013). Theoretical proposal in bullying research: a review. Anales de Psicología, 29, 413-425. doi:10.6018/analesps.29.2.148251

Reniers, Renate L., Corcoran, Rhiannon, Drake, Richard, Shryane, Nick y Völlm, Birgit (2011). The QCAE: A Questionnaire of Cognitive and Affective Empathy. Journal of Personality Assessment, 93, 84-95. doi:10.1080/00223891.2010.528484

Rey, Cesar (2003). La medición de la empatía en preadolescentes varones: adaptación y validación de una escala. Revista Latinoamericana de Psicología, 35(2), 185-194.

Reyes, Isabel. (1993). La redes semánticas naturales, su conceptualización y su utilización en la construcción de instrumentos. Revista de Psicología Social y Personalidad, 9, 8197.

Rogers, Carl. (1966). Psicoterapia centrada en el cliente. Buenos Aires: Paidós.

Spreng R., Nathan, McKinnon, Margaret C., Mar, Raymond A. y Levine, Brian. (2009). The Toronto Empathy Questionnaire: Scale Development and Initial Validation of a FactorAnalytic Solution to Multiple Empathy Measures. Journal of Personality Assessment, 91, 62-71. doi:10.1080/00223890802484381

Stavrinides, Panayiotis, Georgiou, Stelios y Theofanous, Vaso. (2010). Bullying and empathy: a short-term longitudinal investigation. Educational Psychology, 30, 793-802. doi:10.1080/01443410.2010.506004

Sung, Yun y Espegale, Dorothy (2012). A review of research on bullying and peer victimization in school: An ecological system analysis. Aggression and Violent Behavior, 17, 311-322. doi:10.1016/j.avb.2012.03.003 
Swearer, Susan y Hymel, Shelley (2015). Understanding the psychology of bullying. Moving toward a social-ecological diathesis-stress model. American Psychologist, 70, 344-353. doi:10.1037/a0038929

Valdés, Ángel y Carlos, Ernesto (2014). Relación entre el autoconcepto social, el clima familiar y el clima escolar con el bullying en estudiantes de secundarias. Avances en Psicología Latinoamericana, 32, 443-453. doi:10.12804/apl32.03.2014.07

Valdés, Ángel, Carlos, Ernesto, Tánori, Jesús y Madrid, Esthela. (En prensa). Relación entre funcionamiento familiar, emociones morales y violencia entre estudiantes de primaria. Revista Mexicana de Investigación Educativa, 22(71).

Van Noorden, Tirsa, Haselager, Gerbert, Cillessen, Antonius y Bukowski, William. (2014). Empathy and involvement in bullying in children and adolescents: A systematic review. Journal of Youth and Adolescence, 44(3), 637-657. doi:10.1007/s10964-01 40135-6

Volk, Anthony, Dane, Andrew y Marini, Zopito. (2014). What is bullying? A theoretical redefinition. Developmental http://dx.doi.org/10.1016/i.dr.2014.09.001

Review, 34, 327-343.

Vossen, Helen, Piotrowski, Jessica y Valkenburg, Patti. (2015). Development of the adolescent measure of empathy and sympathy. Personality and Individual Differences, 74, 66-71. doi:10.1016/j.paid.2014.09.040 